\title{
Optimization of a Conical Corrugated Antenna Using Multiobjective Heuristics for Radio-Astronomy Applications
}

\author{
S. López-Ruiz, ${ }^{1}$ R. Sánchez Montero, ${ }^{2}$ F. Tercero-Martínez, \\ P. L. López-Espí, ${ }^{2}$ and J. A. López-Fernandez ${ }^{1}$ \\ ${ }^{1}$ Centro Astronómico de Yebes, Instituto Geográfico Nacional, 19141 Yebes, Spain \\ ${ }^{2}$ Signal Theory and Communications Department, University of Alcalá, 28805 Alcalá de Henares, Spain \\ Correspondence should be addressed to P. L. López-Espí; pablo.lopez@uah.es
}

Received 5 May 2016; Accepted 4 July 2016

Academic Editor: Sotirios K. Goudos

Copyright (C) 2016 S. López-Ruiz et al. This is an open access article distributed under the Creative Commons Attribution License, which permits unrestricted use, distribution, and reproduction in any medium, provided the original work is properly cited.

This paper presents the design of a tree sections corrugated horn antenna with a modified linear profile, using NURBS, suitable for radio-astronomy applications. The operating band ranges from 4.5 to $8.8 \mathrm{GHz}$. The aperture efficiency is higher than $84 \%$ and the return losses are greater than $20 \mathrm{~dB}$ in the whole bandwidth. The antenna optimization has been carried out with multiobjective versions of an evolutionary algorithm (EA) and a particle swarm optimization (PSO) algorithm. We show that both techniques provide good antenna design, but the experience carried out shows that the results of the evolutionary algorithm outperform the particle swarm results.

\section{Introduction}

In the recent years, the study of the heavenly body radiation in radioelectric field has been very relevant and has allowed obtaining geodesic information, such as tectonics plaque drift, poles movement, or definition of international reference system used in positioning systems. Signal reception is done by radiotelescopes, which are based on parabolic antennas with a huge dimension. One of the most critical points in the radiotelescope design is the implementation of the parabolic antenna feed. This component mainly determines the frequency range and other properties of the receiver.

For this reason, in 2015, Latvian government decided to change radically the radiotelescope located in Ventspils (Latvia) [1]. This conversion implied the replacement of the optical system (as Cassegrain) by a dual optical with a shaped reflector. The main purpose of this restructuring is to be able to analyze signals between 4.5 and $8.8 \mathrm{GHz}$ to cover the $3.6 \mathrm{~cm}, 5 \mathrm{~cm}$, and $6 \mathrm{~cm}$ radio-astronomy bands, using a single receptor in the antenna feed.

In this work, we have proposed an optimized horn antenna to feed the higher parabolic antenna which was already designed. This prototype is based on a corrugated horn antenna whose profile has been optimized using two softcomputing multiobjective algorithms. On one hand, we have focused on the performance of the evolutionary algorithm (EA) approach to the optimization of the horn antenna. On the other hand, the prototype antenna has been optimized using a particle swarm optimization (PSO) algorithm.

The antenna design involves more than one objective function that must be satisfied according to the initial antenna design specifications [2]. Moreover, these objective functions are conflicting with each other, and consequently no universally optimal solution for the problem can be identified a priori. Therefore, a multiobjective evolutionary algorithm (MOEA) may be applied in order to estimate the nondominated solutions that represent the best possible compromises between the different objectives. Specifically, we have proposed the use of two MOEA techniques, which have been successfully applied recently to a number of difficult optimization problems with excellent results. The first one is based on EA using NSGA-II [3] and the second one combines the NSGA-II operations with a nondominated sorting PSO (NSPSO) [4].

The comparison of both optimization methods using multiobjective algorithm during the antenna design has been 
done. The antenna structure and the optimization method are fully described in this work, including the algorithm's encoding, structure, and objective functions. The optimization algorithms have been programmed in MATLAB and the performance of the antenna has been evaluated using AXIAL modal matching simulator [5].

The rest of the paper is organized as follows. Section 2 describes the antenna structure and its shape constraints. Section 3 describes the multiobjective approach used as optimization method, paying special attention to the objective functions. Next, a detailed discussion of the simulation and measurement results obtained is given in Section 4. Finally, conclusions are summarized in Section 5.

\section{Antenna Description}

The dual-reflector antenna consists of two reflectors and a feed antenna. The feed is conveniently located in a feed cone at the precise distance between the apex of the main reflector and the subreflector in focal point of the reflector antenna.

Many factors must be taken into account when the feed system is designed in order to improve the aperture efficiency of the shaped reflector. These factors can be summarized by the following:

(i) Radiation pattern at $17^{\circ}$ which must be between $-11 \mathrm{~dB}$ and $-14 \mathrm{~dB}$.

(ii) Feeder phase centre stability throughout the full band.

(iii) Low SWR level. The feed is the first passive component of a cryogenic receiver which also has a polarizer and LNA and this value has influence on the system noise figure.

(iv) Good symmetry beam to avoid increasing crosspolarization.

The classical solution is based on a corrugated conical feeder, as it provides beam symmetry and good adaptation to the waveguide. Corrugated horns design begins with the set of specifications that must be accomplished and by the selection of the geometry. There are four main parts in the structure of a corrugated horn:

(1) The aperture, which controls the width of the radiation beam.

(2) The flare angle, which also controls the radiation pattern and the length of the horn.

(3) The geometry of the corrugations, which controls the cross-polar component.

(4) The input waveguide, which controls the input impedance and the mode conversion between a flat and a corrugated guide.

The design of corrugated classic horns is based on controlling the modes that excite $\mathrm{HE}_{11}$ mode. This mode consists of a corresponding combination of $85 \%$ of $\mathrm{TE}_{11}$ mode and $15 \%$ of $\mathrm{TM}_{11}$ mode, with an appropriate relative phase shift between them [6]. $\mathrm{TE}_{11}$ is usually the initial mode in a circular guide, and by an appropriate growth angle of the guide, it is possible to excite $\mathrm{TM}_{11}$ mode with proper amplitude and phase.

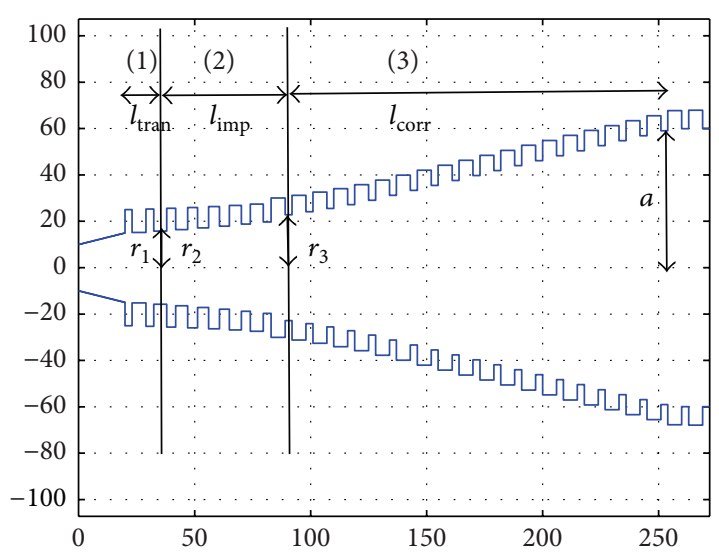

Figure 1: Corrugated horn antenna sections.

The proposed horn is divided into three parts: the initial transition (1), the throat region (2), and the output region (3). The initial transition is used to widen the input guide and does not have corrugations. The throat region has smoothly variable depth and slot corrugations. This region mainly defines the input impedance (reflection losses or SWR value). The output region (3), where the corrugation depth and slot are constant, will define the cross-polar component (Figure 1).

Usually, $\mathrm{TE}_{11}$ mode is excited at the entrance of a circular horn, and this defines the radius $\left(r_{1}\right)$ of the input circular guide according to the classical solutions of the Bessel equations for the wave propagation in that kind of structure. To minimize reflection losses, the depth of the corrugations in the second section should be approximately $\lambda / 2$, where $\lambda$ is the wavelength at the operating frequency, but the depth of the corrugations should be approximately $\lambda / 4$ to excite $\mathrm{HE}_{11}$ mode. Therefore, an adaptation of the depth of the corrugations must be made to meet both criteria. In addition, the size of the corrugations must be variable to increase the operating bandwidth of the antenna. This transition follows a linear change for a smooth transformation [7]. This is observed in Figure 1 and a detailed description of the corrugation design parameters is given in Figure 2.

In addition to Figure 2, another parameter to be chosen is the profile of the horn. The simplest profile is the linear one, as it is the easiest to build and to optimize. However, choosing another profile allows modifying the modal composition in the aperture. This is the way to obtain the required radiation pattern.

The profile variations allow dealing with

(1) restrictions on the radiation pattern, for example, constant taper and symmetry throughout the band,

(2) reducing the input reflections due to discontinuities along the horn,

(3) stability of the phase centre for proper illumination of the reflector antennas throughout the operating band, 


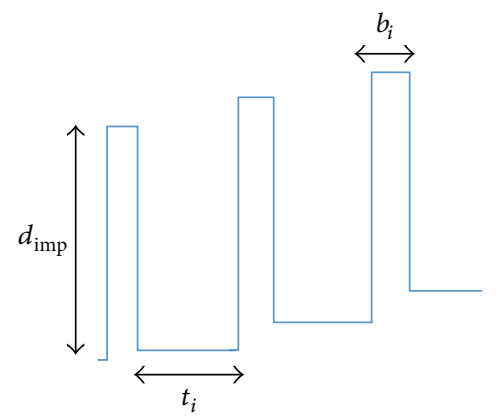

(a)

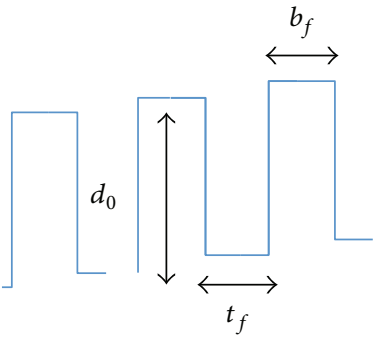

(b)

FIGURE 2: Specific corrugation dimensions. (a) Section (2) of Figure 1. (b) Section (3) of Figure 1.

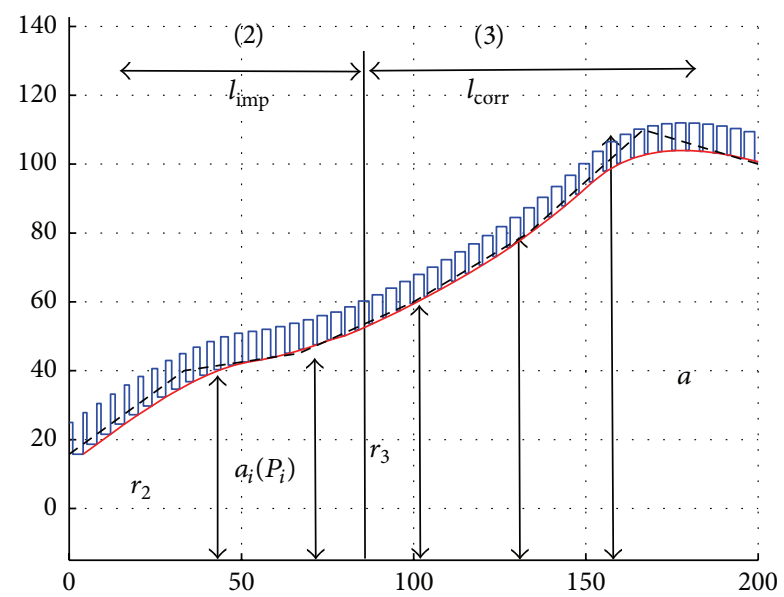

FIGURE 3: Profile horn defined by NURBS curve with five control points.

(4) reducing the size of the horn to facilitate the design of the cryogenic receiver.

Profiles can be derived from analytical formulas $[8,9]$ or can be defined by a limited set of points that define curves (i.e., splines). Parametric profiles are defined by a small set of parameters. The advantage of these curves is that they can approach any analytical curve; thus the optimization process is not restricted to using one specific type of profile [10]. Among these kinds of parametric profile curves are NURBS (nonuniform B-splines) [11], which are widely used in models of graphic surfaces. NURBS are defined by a set of ordered points $P_{i}=0,1, \ldots, n$, defined as "Control Polygon." A fivepoint NURB profile has been chosen for this research.

Finally, the designed horn is defined by a list of parameters. In our case, we have configured the horn with the variables shown in Table 1 and previously detailed in Figures 1,2 , and 3 .

The variables defined in Table 1 should have a lower limit and an upper limit to meet electromagnetic conditions described previously. For example, $r_{1}$ defines the cutoff frequency of the input waveguide and therefore the operating
TABLE 1: Dimensions limits.

\begin{tabular}{lcc}
\hline Parameter $(\mathrm{mm})$ & Lower limit $(\mathrm{mm})$ & Upper limit $(\mathrm{mm})$ \\
\hline$r_{1}$ & 30 & 36 \\
$l_{\text {tran }}$ & 0 & 30 \\
$r_{2}$ & 30 & 55 \\
$d_{\text {imp }}$ & 10 & 24 \\
$t_{i}$ & 5 & 11 \\
$b_{i}$ & 1 & 4 \\
$r_{3}$ & 60 & 90 \\
$d_{0}$ & 10 & 28 \\
$t_{f}$ & 2.5 & 5.5 \\
$b_{f}$ & 3 & 11.5 \\
$l_{p}$ & 400 & 680 \\
$a$ & 180 & 300 \\
$a_{1}$ & 30 & 80 \\
$a_{2}$ & 70 & 120 \\
$a_{3}$ & 90 & 140 \\
$a_{4}$ & 120 & 200 \\
$a_{5}$ & 140 & 280 \\
\hline
\end{tabular}

band; the corrugations are chosen to have an approximate value of $\lambda / 2$ in the first section and $\lambda / 4$ in the following ones. The aperture value, $a$, determines the external dimension of the horn. Finally, the values of the parametric profile are selected to have values between $r_{1}$ and $a$.

Additionally, another set of constraints must be applied to make the horn mechanically buildable. These are given in Table 2 .

\section{Algorithms' Description}

The huge number of design parameters to be satisfied and the constraints of the horn antenna geometry, specified in Table 2, make it really difficult to obtain an exact expression based on design equations. For this reason, it is necessary to employ an automatic method to help us achieve the antenna design. Optimization algorithms are introduced to accomplish the requirements of the antenna in terms of performance. In 
TABLE 2: Constraints in the antenna geometry.

\begin{tabular}{lc}
\hline$r_{2}>r_{1}$ & $a>a_{5}$ \\
$r_{3}>r_{2}$ & Integer number of corrugations \\
$a_{1}>r_{2}$ & Variables within the limits \\
$a_{i}+1>a_{i}$ & Rounding wing tenth of a millimeter \\
\hline
\end{tabular}

this context, it is easy to find several works in the literature, where novel metaheuristics approaches, such as evolutionary computation or particle swarm algorithms, are applied to antenna design. Following this rationale, we considered employing the monoobjective metaheuristics algorithm but the horn antenna did not satisfy the initial specification. To this end, it is deemed reasonable to implement a multiobjective optimization algorithm with several objective functions, similarly to what has been recently done in [12] for planar antenna array.

\subsection{Nondominated Sorting Genetic Algorithm-II (NSGA-II)} Description. The use of evolutionary algorithms for optimization purposes has gained momentum in the last years, being thoroughly applied to distinct fields ranging from engineering and computer science to medicine and economics [13]. On the other hand, the potential of evolutionary algorithms for solving multiobjective optimization problems was first discussed in [14]. Since then, this area of research has attracted an ever-growing interest within the scientific community [15-17], including their application to the optimal design of antennas and other radiant devices [18-20]. There are two main reasons for applying this kind of algorithms to multiobjective optimization problems. First, their population-based nature makes them suitable for finding several members of the Pareto-optimal set in a single run of the algorithm, instead of having to perform separate runs as done in traditional programming techniques [21]. Second, they are usually applied to continuous optimization problems being able to easily deal with discontinuous and concave Pareto fronts.

Evolutionary algorithms (EAs) are robust problem solving techniques based on natural evolution processes. They are population-based techniques which codify a set of possible solutions to the problem and evolve it through the application of the so-called evolutionary operators [22, 23]. Among EAs, evolutionary programming (EP) approaches are usually applied to continuous optimization problems. This algorithm is characterized by using mutation, crossover, and selection operators. Several versions of the algorithm have been proposed in the literature. The classical evolutionary programming (CEP) algorithm was first described by Bäck and Schwefel in [24]. It is used to optimize a given function $f(x)$ which is obtaining $x_{0}$ such that $f\left(x_{0}\right)<f(x), x \in$ [lim_inf, lim_sup]. The CEP algorithm performs as follows:

(1) Generate an initial population of $\mu$ individuals (solutions). Let $t$ be a counter for the number of generations; set it to $t=1$. Each individual is taken as a pair of real-valued vectors $\left(x_{i}, \sigma_{i}\right), \forall i \in\{1, \ldots, \mu\}$, where $x_{i}^{\prime}$ s are objective variables and $\sigma_{i}^{\prime}$ s are standard deviations for Gaussian mutations.

(2) Evaluate the fitness value for each individual $\left(x_{i}, \sigma_{i}\right)$ using the problem's objective function.

(3) Each parent $\left(x_{i}, \sigma_{i}\right),\{i=1, \ldots, \mu\}$ then creates a single offspring $\left(x_{i}^{\prime}, \sigma_{i}^{\prime}\right)$ as follows:

$$
\begin{aligned}
& x_{i}^{\prime}=x_{i}+\sigma_{i} \cdot N_{1}(0,1), \\
& \sigma_{i}^{\prime}=\sigma_{i} * \exp \left(\tau^{\prime} \cdot N(0,1)+\tau \cdot N(0,1)\right),
\end{aligned}
$$

where $N(0,1)$ denotes a normally distributed onedimensional random number with mean of zero and standard deviation of one and $N(0,1)$ and $N_{1}(0,1)$ are vectors containing random numbers of mean of zero and standard deviation of one, generated anew for each value of $i$. The parameters $\tau$ and $\tau^{\prime}$ are commonly set to $(\sqrt{2 \sqrt{n}})^{-1}$ and $(\sqrt{2 n})^{-1}$, respectively [22], where $n$ is the length of the individuals.

(4) If $x_{i}(j)>$ lim_sup then $x_{i}(j)=$ lim_sup and if $x_{i}(j)<$ lim_inf then $x_{i}(j)=$ lim_inf.

(5) Calculate the fitness values associated with each offspring $\left(x_{i}^{\prime}, \sigma_{i}^{\prime}\right) \forall i \in\{1, \ldots, \mu\}$.

(6) Conduct pairwise comparison over the union of parents and offspring: for each individual, $P$ opponents are chosen uniformly at random from all the parents and offspring. For each comparison, if the individual's fitness is better than the opponent's, it receives a "win."

(7) Select $\mu$ individuals out of the union of parents and offspring that have the most "wins" to be parents of the next generation.

(8) Stop if the halting criterion is satisfied, and if not, set $t=t+1$ and go to Step (3).

The easy way to get MOA based on evolutionary programming is to use a popular nondomination based genetic algorithm for multiobjective optimization (NSGA) [25]. It is a very effective algorithm but has been generally criticized for its computational complexity, for lack of elitism, and for choosing the optimal parameter value for sharing parameter $\sigma_{\text {share }}$. A modified version, NSGA-II [3], was developed, which has a better sorting algorithm and incorporates elitism, and no sharing parameter needs to be previously chosen. According to Deb et al. [3], the previously described EA algorithm needs to be modified and performs as follows:

(1) Create a random parent population.

(2) Sort the population on a nondominated basis.

(3) Carry out tournament selection.

(4) Create offspring using recombination and mutation.

(5) Combine parent and offspring. 
(6) Sort the population on a nondominated basis.

(7) Emphasize the best nondominated solutions for the different fronts.

(8) Choose the best $N$ solutions according to their crowding distance (a measure of the density solutions in the neighbourhood).

(9) Increase the generation counter. Stop if the halting criterion is satisfied, and if not, go to Step (3).

As can be seen, MOEA can be considered an extension of the EA which maintains a diverse set of solutions moving toward the Pareto-optimal region.

\subsection{Nondominated Sorting Particle Swarm Optimization} (NSPSO) Description. Particle swarm optimization (PSO) is a population-based stochastic optimization technique developed by Kennedy and Eberhart [26], inspired by social behavior of bird flocking and fish schooling. This algorithm has already been applied to antenna optimization problems $[27,28]$. A PSO system is initialized with a population of random solutions and searches for the optimal one by updating generations. PSO has no evolution operators such as crossover and mutation as genetic algorithms do but has potential solutions instead, called particles, which fly through the problem search space to look for promising regions according to its own experiences and experiences of the whole group. Thus, social information is shared, and individuals profit from the discoveries and previous experiences of other particles in the search. The PSO is considered a global search algorithm. Mathematically, given a swarm of $N$ particles, each particle $i \in\{1,2, \ldots, N\}$ is associated with a position vector $x_{i}=\left(x_{1}^{i}, x_{2}^{i}, \ldots, x_{K}^{i}\right)$ with $K$ being the number of parameters to be optimized in the problem. Let $p_{i}$ be the best previous position that particle $i$ has ever found; that is, $p_{i}=\left(p_{1}^{i}, p_{2}^{i}, \ldots, p_{K}^{i}\right)$, and let $g$ be the group's best position (leader) ever found by the algorithm; that is, $g=\left(g_{1}, g_{2}, \ldots, g_{K}\right)$. At each iteration step $k+1$, the position vector of the $i$ th particle is updated by adding an increment vector $\Delta x_{i}(k+1)$, called velocity $v_{i}(k+1)$, as follows:

$$
\begin{aligned}
v_{d}^{i}(k+1)= & v_{d}^{i}(k)+c_{1} r_{1}\left(p_{d}^{i}-x_{d}^{i}(k)\right) \\
& +c_{2} r_{2}\left(g_{d}-x_{d}^{i}(k)\right), \\
v_{d}^{i}(k+1)= & \frac{v_{d}^{i}(k+1) \cdot v_{d}^{\max }}{\left|v_{d}^{i}(k+1)\right|}, \text { if }\left|v_{d}^{i}(k+1)\right|>v_{d}^{\max }, \\
x_{d}^{i}(k+1)= & x_{d}^{i}(k)+v_{d}^{i}(k+1),
\end{aligned}
$$

where $c_{1}$ and $c_{2}$ are two positive constants, $r_{1}$ and $r_{2}$ are two random parameters which are found uniformly within the interval $[0,1]$, and $v_{d}^{\max }$ is a parameter that limits the velocity of the particle in the $i$ th coordinate direction. This iterative process will continue until a stop criterion is satisfied, and this forms the basic iterative process of a standard PSO algorithm [26].

As stated earlier, there are two approaches to solve the MOA based on PSO algorithm. One approach is the classical weighted-sum approach, where the objective function is formulated as a weighted sum of the objectives. But the problem lies in the correct selection of the weights or utility functions to characterize the decision makers preferences. The second approach called Pareto-optimal (NSPSO) solution has no unique or perfect solution, but a set of nondominated, alternative solutions, known as the Pareto-optimal set [4], is achieved. A good description of the MO-PSO algorithm is given in [29]:

(1) Initialize swarm.

(2) Initialize leaders in an external file with the best nondominated particles.

(3) Measure quality of the leaders.

(4) For each particle, select leader and update its position using velocity.

(5) Evaluate the particle.

(6) Update the leaders in the external archive.

(7) Measure the quality of the leaders.

(8) Increase the generation counter. Stop if the halting criterion is satisfied, and if not, go to Step (4).

The selection of the leader is a key component. The most straightforward approach is to consider every nondominated solution as a new leader and then just one leader has to be selected.

3.3. Fitness Function Definition. In particular, the antenna parameters' optimization problem initially described in Section 2 is composed of several fitness functions that must be simultaneously satisfied. These are described below:

(1) Radiation pattern $\left(\operatorname{Horn} E_{\theta}\right)$ at specific direction $(\theta)$, called $(T(\mathrm{~dB}))$, in the whole bandwidth must satisfy the following:

$$
f(1)= \begin{cases}0 & \max \left(\left|\operatorname{Horn} E_{\theta}^{4.5-8.8 \mathrm{GHz}}-T(\mathrm{~dB})\right|\right)<x_{1} \\ \max \left(\left|\operatorname{Horn} E_{\theta}^{4.5-8.8 \mathrm{GHz}}-T(\mathrm{~dB})\right|\right) & x_{1} \leq \max \left(\left|\operatorname{Horn} E_{\theta}^{4.5-8.8 \mathrm{GHz}}-T(\mathrm{~dB})\right|\right) \leq x_{2} .\end{cases}
$$


(2) Second objective function is related to cross-polar polarization (XPpeak), which must be less than a specific value named $T^{\prime}$ :

$$
f(2)=\left\{\begin{array}{l}
0 \\
\max \left(\left|\mathrm{XPpeak}^{4.5-8.8 \mathrm{GHz}}-T^{\prime}(\mathrm{dB})\right|\right)
\end{array}\right.
$$

(3) Next objective is that the return losses $\left(S_{11}\right)$ of the design antenna must be less than a specific value given by variable $T^{\prime \prime}$ :

$$
f(3)=\left\{\begin{array}{l}
0 \\
\max \left(\left|S_{11}^{4.5-8.8 \mathrm{GHz}}-T^{\prime \prime}(\mathrm{dB})\right|\right)
\end{array}\right.
$$

(4) Antenna phase centre has to be stable in all working bandwidth. So, first of all, it is necessary to calculate the phase centre at a specific direction $(\theta)$ of the radiation pattern $\left(\mathrm{PhHorn} E_{\theta}\right)$ in the whole bandwidth [30]:

$$
\mathrm{zpc}=\lambda \cdot \frac{\operatorname{PhHorn} E_{\theta=0}^{4.5-8.8 \mathrm{GHz}}-\operatorname{PhHorn} E_{\theta}^{4.5-8.8 \mathrm{GHz}}}{360 \cdot(1-\cos (\theta))} .
$$

Next, a linear regression must be calculated to determine the slope of the phase centre. If the linear regression error is very high, the solution must be rejected:

$$
f(4)= \begin{cases}0 & \text { slope }<x_{1}^{i i i} \\ \text { slope } & x_{1}^{i i i} \leq \text { slope } \leq x_{2}^{i i i} .\end{cases}
$$

(5) Radiation pattern ( $E$-plane and $H$-plane) must be symmetrical between 0 and a particular direction $\theta$ :

$$
\operatorname{dif}=\sqrt{\sum_{f=4.5}^{f=8.8} \sum_{\theta=0}^{\theta} \frac{1}{\text { no. freq } \cdot \text { no. } \theta} \mid H \text { Plane }-E \text { Plane }\left.\right|^{2}},
$$

where no. freq is the number of simulated frequencies and no. $\theta$ is the number of points in $\theta$-axis:

$$
f(5)= \begin{cases}0 & \text { dif }<x_{1}^{i v} \\ \operatorname{dif} & x_{1}^{i v} \leq \operatorname{dif} \leq x_{2}^{i v} .\end{cases}
$$

When the simulation is finished, the solutions are organized to determine the best result. This has been done by calculation $\max \left(\left|S_{11}^{4.5-8.8 \mathrm{GHz}}-T^{\prime \prime}(\mathrm{dB})\right|\right)<x_{1}^{\prime \prime}$
$x_{1}^{\prime \prime} \leq \max \left(\left|S_{11}^{4.5-8.8 \mathrm{GHz}}-T^{\prime \prime}(\mathrm{dB})\right|\right) \leq x_{2}^{\prime \prime}$.

$$
\begin{aligned}
& \max \left(\mid \text {XPpeak}^{4.5-8.8 \mathrm{GHz}}-T^{\prime}(\mathrm{dB}) \mid\right)<x_{1}^{\prime} \\
& x_{1}^{\prime} \leq \max \left(\mid \text { XPpeak }^{4.5-8.8 \mathrm{GHz}}-T^{\prime}(\mathrm{dB}) \mid\right) \leq x_{2}^{\prime} .
\end{aligned}
$$

of the function value $\varepsilon^{k}$ of $i$ th objective function $F_{i}$ for the solution $k$, defined as follows:

$$
\begin{aligned}
& \varepsilon_{i}^{k} \\
& \quad= \begin{cases}1 & f(i)<f(i)^{\min } \\
\frac{f(i)^{\max }-f(i)}{f(i)^{\max }-f(i)^{\min }} & f(i)^{\min } \leq f(i) \leq f(i)^{\max } \\
0 & f(i)>f(i)^{\max },\end{cases}
\end{aligned}
$$

where $f(i)^{\max }$ and $f(i)^{\min }$ are the maximum and minimum of $i$ th objective function among all nondominated solutions, respectively. The above equation provides a measure of the degree of satisfaction of each objective function for a particular solution.

The corresponding membership function $\varepsilon^{k}$ for each solution $k$ in Pareto solution set is calculated as follows:

$$
\begin{aligned}
\varepsilon^{k} & =\frac{\sum_{i=1}^{N} \varepsilon_{i}^{k}}{\sum_{k=1}^{M} \sum_{i=1}^{N} \varepsilon_{i}^{k}}, \\
\varepsilon_{\text {norm }}^{k} & =\frac{\varepsilon^{k}-\min \left(\varepsilon^{k}\right)}{\max \left(\varepsilon^{k}\right)-\min \left(\varepsilon^{k}\right)},
\end{aligned}
$$

where $M$ is the number of Pareto solutions and $N$ is the number of optimization objectives. Finally, it is normalized using the value $\varepsilon^{k}$ obtained, so the values are ranged within $[0,1]$ and the best compromised solution is the one achieving the maximum of $\varepsilon^{k}$.

3.4. Connection between Simulator and Optimization Algorithms. As we have mentioned before, the synthesis of dualreflector systems is an advanced topic and many factors must be taken into account when shaped reflectors have to be designed for improved aperture efficiency. The optimization 


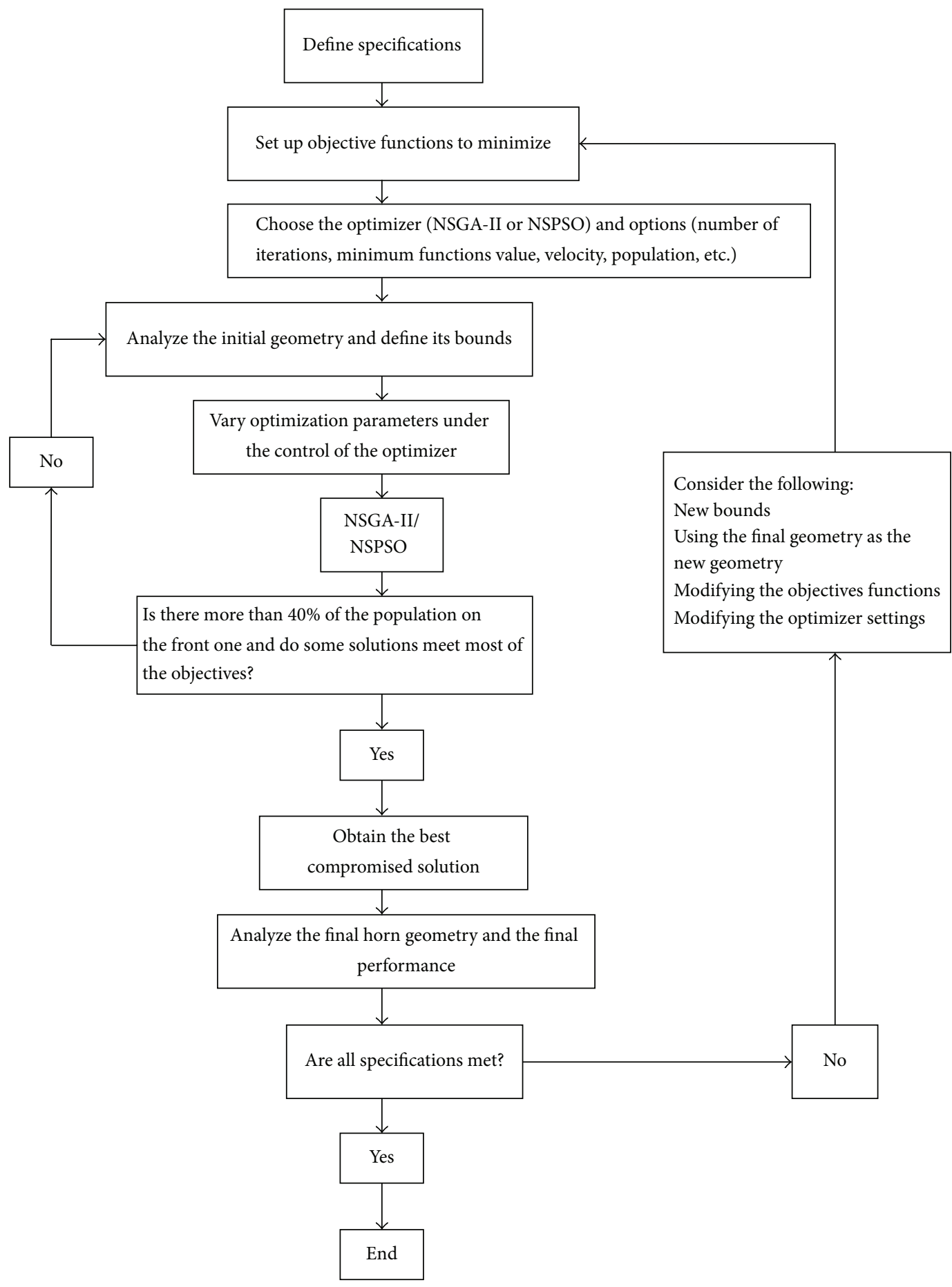

FIGURE 4: Flow graph of horn optimization.

can be carried out using the algorithms described in Section 3 taking into account the limits and constraints given in Section 2.

Both optimization algorithms have been programmed in MATLAB, whereas the antenna simulation has been performed using AXIAL simulation software [10], which consists of mode matching analysis software of mixed circular and coaxial waveguide structures.
The connection between MATLAB and AXIAL has been done as follows: first, the multiobjective algorithms, detailed in Section 3, generate the set (population) of variables (individuals) to be optimized $\left\{r_{1}, l_{\text {tran }}, r_{2}, d_{\text {imp }}, t_{i}, b_{i}\right.$, $\left.r_{3}, d_{0}, t_{f}, b_{f}, l_{p}, a, a_{1}, a_{2}, a_{3}, a_{4}, a_{5}\right\}$ by using MATLAB, which are next saved in a plane text file. These stored values are retrieved by AXIAL and the simulation of the horn is carried out. When this simulation is done, the results of radiation 


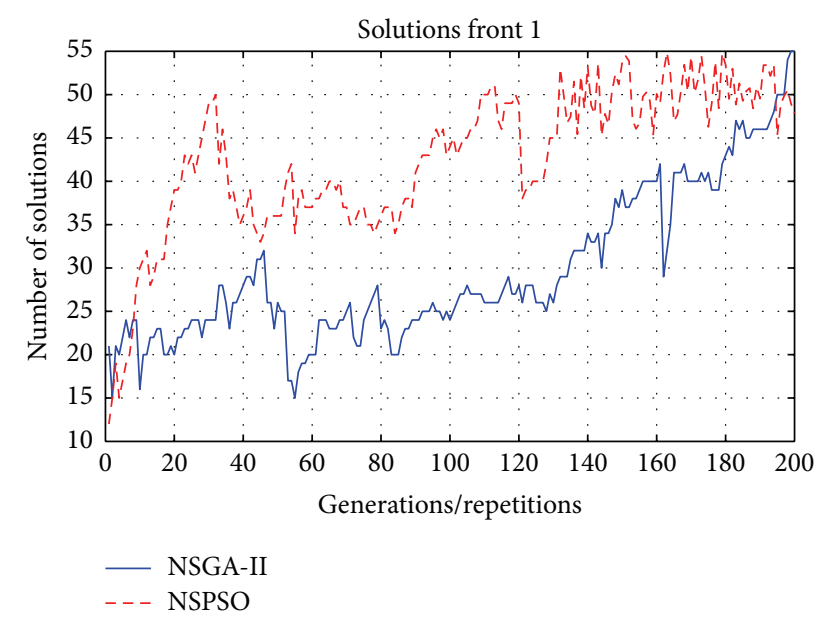

FIGURE 5: Number of solutions obtained with the NSGA and NSPSO approaches.

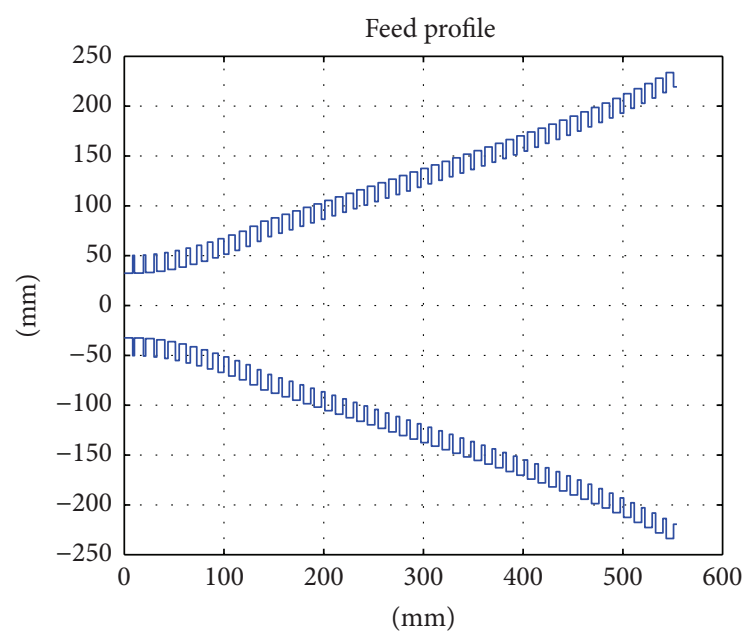

Figure 6: Profile of the final horn.

TABLE 3: Goal values of the fitness function.

\begin{tabular}{lcccc}
\hline Fitness function & $\theta(\mathrm{deg})$ & $T(\mathrm{~dB})$ & $x_{1}$ & $x_{2}$ \\
\hline$f(1)$ & 17 & -12.5 & 1.5 & 2.5 \\
$f(2)$ & - & -20 & 1 & 10 \\
$f(3)$ & - & -30 & 1 & 15 \\
$f(4)$ & 10 & - & 10 & 80 \\
$f(5)$ & 20 & - & 0.5 & 2.5 \\
\hline
\end{tabular}

pattern at $\theta$ direction $\left(\operatorname{Horn} E_{\theta}\right)$, cross-polar polarization (XPpeak), reflection coefficient $\left(S_{11}\right)$, antenna phase centre $\left(\operatorname{PhHorn} E_{\theta}\right)$, and $E$-plane and $H$-plane radiation pattern are stored in a new text file. Finally, MATLAB gets these values, and the different fitness functions of the individual are computed and evaluated according to the definitions given in Section 3.3 and using the values indicated in Table 3. The described process is repeated for each individual until the entire population is evaluated.
Figure 4 represents the flow graph used for optimization of the horn; the block of the algorithm corresponds to NSGAII or NSPSO.

As the radiation pattern at $17^{\circ}$ must be between $-11 \mathrm{~dB}$ and $-14 \mathrm{~dB}$, if the value $f(1)$ is less than $1.5\left(x_{1}=14-12.5=1.5\right.$ or $\left.x_{1}=12.5-11=1.5\right)$ the horn meets the specifications and if the maximum difference is greater than $1 \mathrm{~dB}\left(x_{2}=12.5-\right.$ $11-1=2.5$ or $\left.x_{2}=14+1-12.5=2.5\right)$ at any frequency the solution is discarded.

A low cross-polar is also important, $f(2)$; we define a good cross-polar value between $-10 \mathrm{~dB}$ and $-20 \mathrm{~dB}\left(x_{2}=\right.$ $20-10=10)$ and a very good cross-polar value is less than $-19 \mathrm{~dB}\left(x_{1}=20-10=1\right)$ at any frequency; if cross-polar value is greater than $-10 \mathrm{~dB}$ at any frequency the solution is discarded.

For the return losses, $f(3)$, we define a good $S_{11}$ value between $-15 \mathrm{~dB}$ and $-30 \mathrm{~dB}\left(x_{2}=30-15=15\right)$ and a very good $S_{11}$ if it is less than $-29 \mathrm{~dB}\left(x_{1}=30-29=1\right)$ at any frequency; if $S_{11}$ is greater than $-15 \mathrm{~dB}$ at any frequency, the solution is discarded.

Next, $f(4)$ calculates the slope of the phase centre. If we represent the phase centre in $X Y$ diagram, where $x$-axis is the frequency and $y$-axis is the distance, the slope could be related to the following:

$$
\begin{aligned}
\text { slope } & =\frac{\mathrm{zpc}_{1}-\mathrm{zpc}_{0}}{f_{1}-f_{0}}=\frac{\mathrm{zpc}_{8.8}(\mathrm{~mm})-\mathrm{zpc}_{4.5}(\mathrm{~mm})}{8.8-4.5} \\
& =\frac{\Delta \mathrm{zpc}(\mathrm{mm})}{4.3} .
\end{aligned}
$$

Therefore, we have calculated $x_{1}$ and $x_{2}$ for the maximum variation, as phase centre variation is ranged between $43 \mathrm{~mm}$ $\left(x_{1}=43 / 4.3=10\right)$ and $344 \mathrm{~mm}\left(x_{2}=344 / 4.3=80\right)$.

Finally, the radiation pattern must be symmetric, $f(5)$; we define $x_{2}=2.5$ as the maximum difference between radiation patterns because, for greater values, the antenna efficiency decreases. If $f(5)$ is greater than 2.5 at any frequency, the solution is discarded.

\section{Results and Discussion}

Using the previous procedure, we have set a population/ swarm size of 100 chromosomes/particles and a total number of 200 iterations. After that, the final values of the population can be classified according to their $\varepsilon^{k}$ values. The results are given in Table 4 . The best solution was obtained from the genetic algorithm, and also NSGA-II obtained a greater number of valid solutions. On the contrary, as can be seen in Figure 5, PSO solutions reach the Pareto front faster than EP ones which can reduce optimization time but also reduce the diversity of the population and the number of valid solutions.

The final dimensions of the best horn obtained $\left(\max \left(\varepsilon^{k}\right)\right)$ using the two algorithms are shown in Table 5.

The resulting profile shown in Figure 6 is quite similar to a linear one, but it is not perfectly linear, and it improves crosspolar and symmetry of the beams.

The results of the radiation pattern are shown in Figure 7. They fulfill the taper specification over the whole bandwidth. Radiation patterns show a symmetrical beam in the whole 

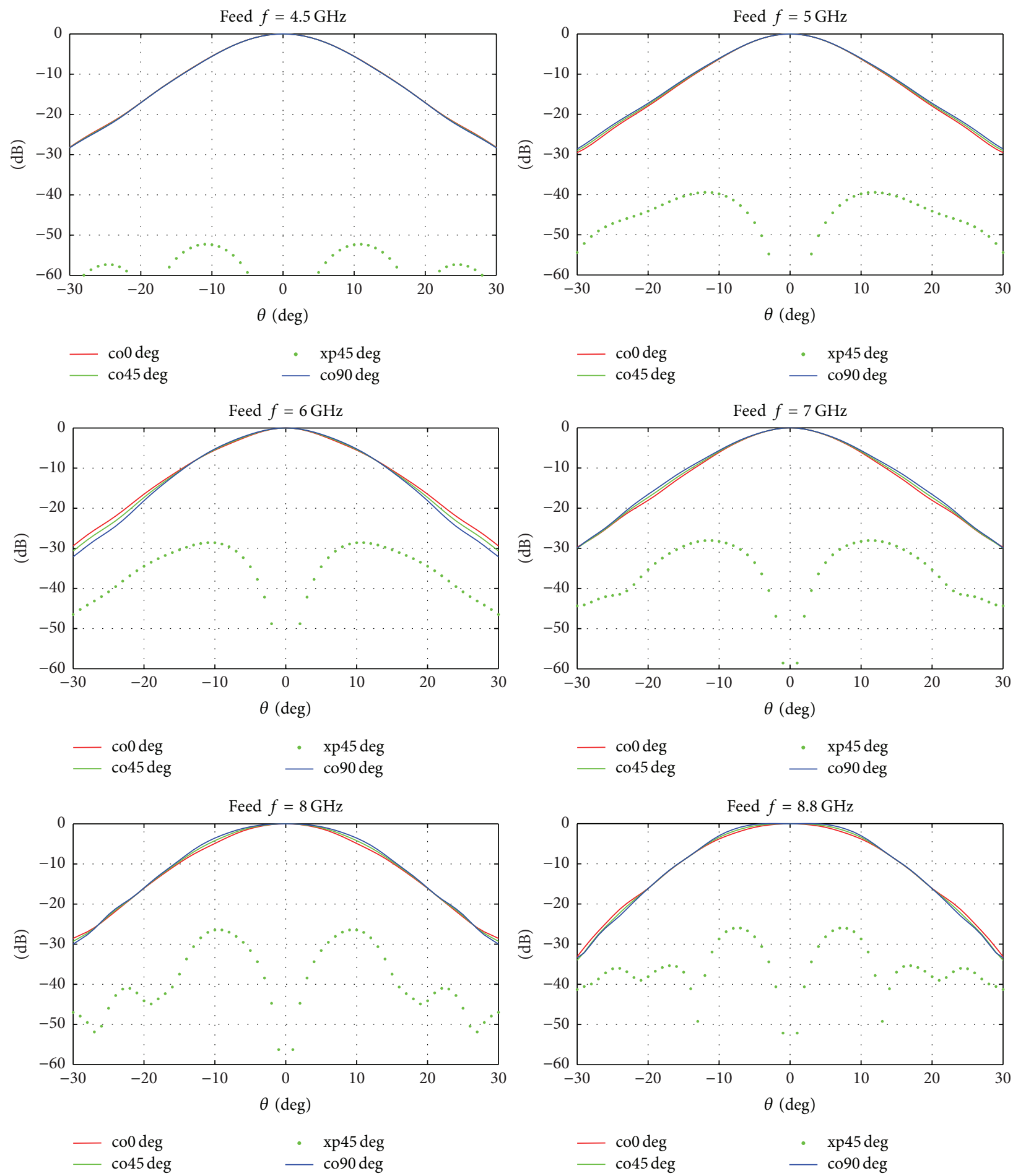

FiguRE 7: Simulated beam pattern of feed (blue-copolar $H$-plane, red-copolar E-plane, green-copolar diagonal plane, and green dot-crosspolar diagonal plane).

bandwidth and the feed asymmetries can be found only in the different side lobe levels.

4.1. Measurements. The simulated horn antenna has been built and finally measured in the anechoic chamber at the Yebes Astronomic Centre [31]. Two different measurements were done: the scattering parameter measurement and the radiation patterns over the frequency range from $4.5 \mathrm{GHz}$ to $8.8 \mathrm{GHz}$.

Two different waveguide standards were used to cover the frequency range adequately: WR159 standard covers $4.90 \mathrm{GHz}$ to $7.05 \mathrm{GHz}$ but it was used from $4.50 \mathrm{GHz}$ and 


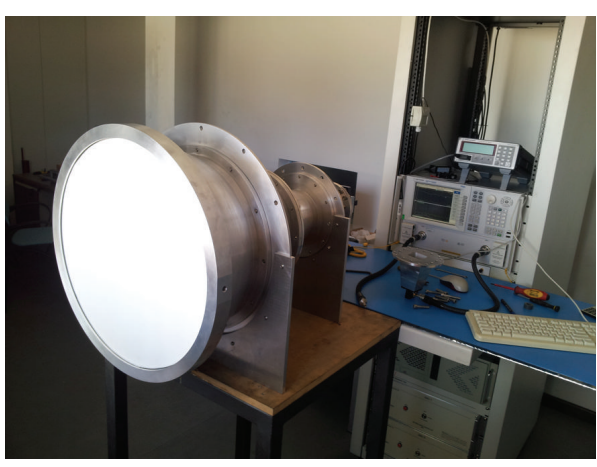

(a)

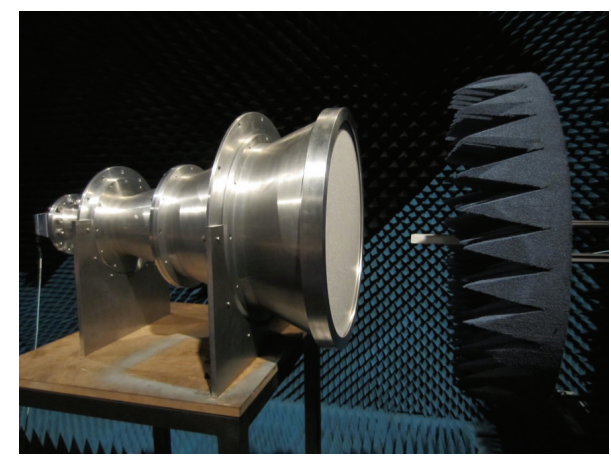

(b)

FIGURE 8: Measured port reflection (a) and radiation pattern (b).

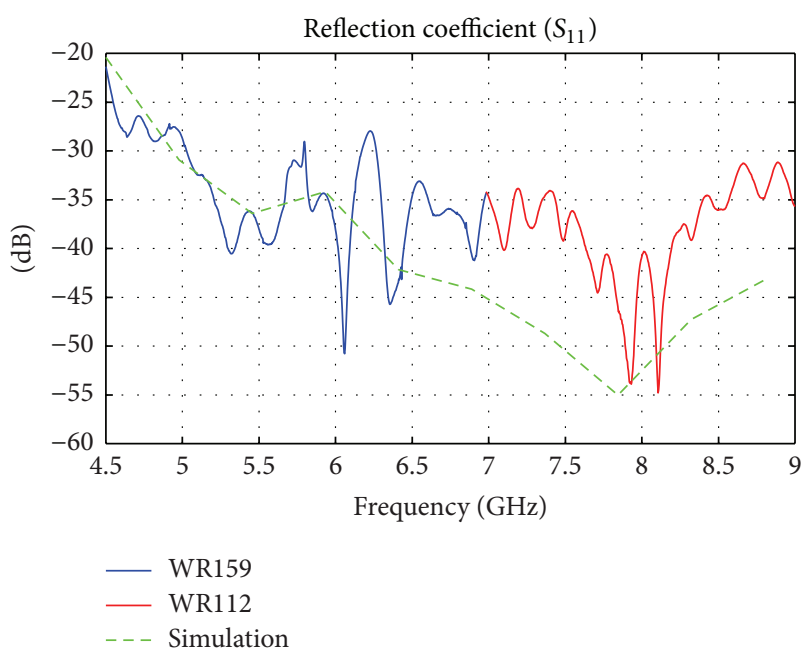

FIGURE 9: Reflection coefficient of the final design.

TABle 4: Percentage of solutions obtained.

\begin{tabular}{lcccc}
\hline Algorithm & $\varepsilon^{k}=1$ & $0.75 \leq \varepsilon^{k}<1$ & $0.5 \leq \varepsilon^{k}<0.75$ & $\varepsilon^{k}<0.50$ \\
\hline NSGA-II & 1 & 34 & 49 & 16 \\
NSPSO & 0 & 9 & 54 & 37 \\
\hline Total & 1 & 43 & 103 & 53 \\
\hline
\end{tabular}

WR112 standard covers $7.05 \mathrm{GHz}$ to $10.0 \mathrm{GHz}$. To measure the scattering parameter $S_{11}$, a microwave absorber is placed in the feed aperture to match it (Figure 8). In Figure 9, the final port reflection coefficient is shown along all the feed bandwidth. It is the final measurement in the $45 \mathrm{~mm}$ squared port, that is, the interface with the cryogenic receiver.

The radiation patterns of the feed were also measured at the Yebes anechoic chamber [31] (Figure 10). The measurements were performed with two setups because of the large bandwidth. Again two families of waveguides and adaptors need to be used. There was one setup to measure from 4.5 to $7.0 \mathrm{GHz}$ and a second one to measure from 7.0 to $8.8 \mathrm{GHz}$. The cross-polar worst level is better than $-20 \mathrm{~dB}$.
TABLE 5: Final values.

\begin{tabular}{lcc}
\hline Parameter $(\mathrm{mm})$ & NSGA-II & NSPSO \\
\hline$r_{1}$ & 32.4 & 35.7 \\
$l_{\text {tran }}$ & 0 & 0 \\
$r_{2}$ & 32.4 & 35.7 \\
$d_{\text {imp }}$ & 18.9 & 18 \\
$t_{i}$ & 9 & 9.1 \\
$b_{i}$ & 1.7 & 2.7 \\
$r_{3}$ & 67.4 & 80.1 \\
$d_{0}$ & 17.2 & 16.5 \\
$t_{f}$ & 3.4 & 3.6 \\
$b_{f}$ & 7.3 & 8.2 \\
$l_{p}$ & 545.7 & 649 \\
$a$ & 219.3 & 273 \\
$a_{1}$ & 47.9 & 40 \\
$a_{2}$ & 100 & 80.3 \\
$a_{3}$ & 138.1 & 120 \\
$a_{4}$ & 163.2 & 150 \\
$a_{5}$ & 185.5 & 187 \\
$f(1)$ & 0 & 4 \\
$f(2)$ & 0 & 0.5 \\
$f(3)$ & 0 & 0 \\
$f(4)$ & 1.7 & 0 \\
$f(5)$ & 60.18 & 74 \\
$\varepsilon^{k}$ & 1 & 0.97 \\
\hline
\end{tabular}

The phase centre is calculated at several frequencies along the feed bandwidth (Figure 11). The predictions about the phase centre in the simulations are confirmed with this measurement. The optimal position of the feed for all frequencies is $660 \mathrm{~mm}$ from the aperture because it is the phase centre position for the highest frequency and it is the more sensitive value to the axial misalignment.

Finally, the efficiency of the aperture is calculated using real measured data. The position of the feed in the antenna reference frame is calculated with the information of the phase centre position measured previously. The feed should 

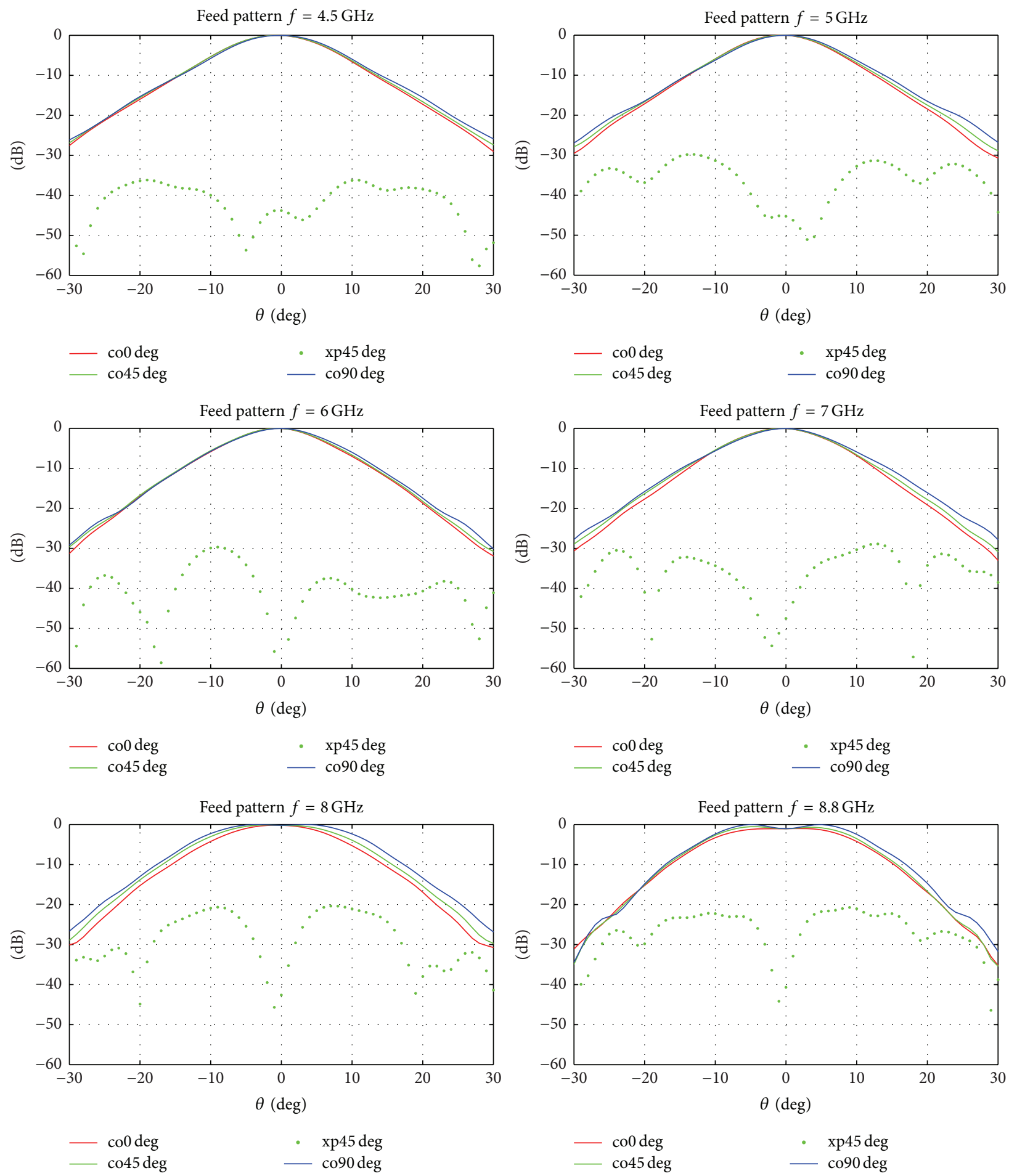

Figure 10: Measured beam pattern of feed (blue-copolar $H$-plane, red-copolar $E$-plane, green-copolar diagonal plane, and green dot-crosspolar diagonal plane).

stand at one position, where all the efficiency of the antenna remains high for all the bandwidth. As can be seen, the results of the physical optics calculation shown in Figure 12 give that the aperture efficiency is higher than $84 \%$ in the whole bandwidth.

\section{Conclusions}

A corrugated horn with modified profile and a matching corrugated section has been proposed. The designed antenna is a tree section one based on a modified linear profile antenna and has been optimized using two different multiobjective 


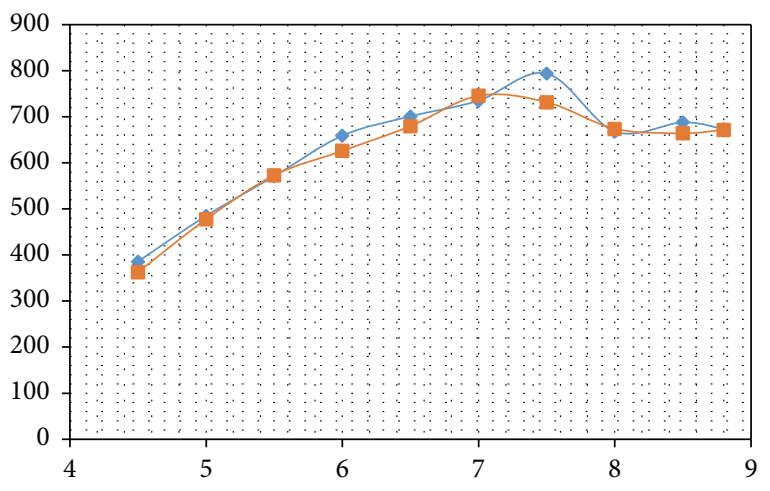

FIGURE 11: Phase centre position of final feed ( $\mathrm{mm}$ from the feed aperture). Measured (blue); simulation (orange).

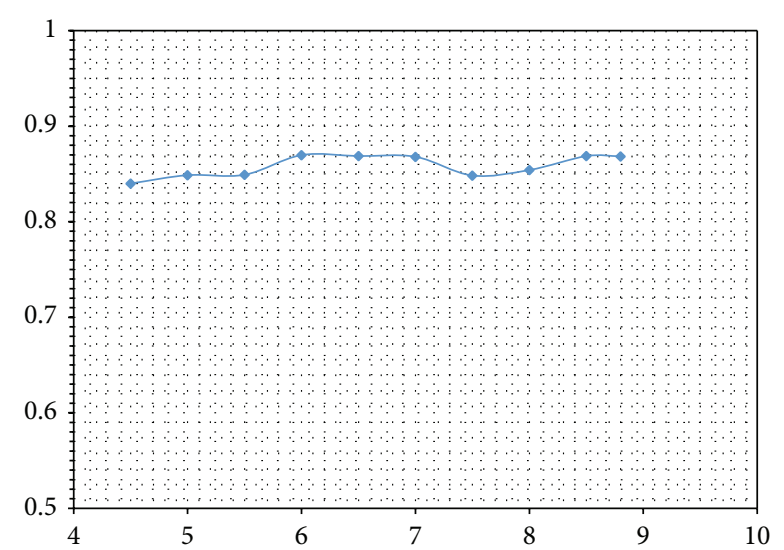

FIGURE 12: Aperture efficiency using the measured data of final feed standing in a fixed position in the antenna. The focal point of the antenna is at $660 \mathrm{~mm}$ from the feed aperture.

algorithms. The best solution was obtained from the genetic algorithm. On the contrary, particle swarm algorithm solutions reach the Pareto front faster than genetic ones.

The algorithms have been programmed in MATLAB and the simulations have been performed with AXIAL. We have set a population/swarm size of 100 chromosomes/particles and a total number of 200 iterations. The results show a good agreement between simulated and measured.

The constructed antenna fulfills all the requirements previously stated: radiation pattern at $17^{\circ}$ which must be between $-11 \mathrm{~dB}$ and $-14 \mathrm{~dB}$, feeder phase centre stability throughout the full frequency band, low SWR level, and good symmetry beam. The aperture efficiency is higher than $84 \%$ in the whole bandwidth.

\section{Notations}

Parameters of the Horn Antenna

$r_{1}$ : Input waveguide radius

$l_{\text {tran }}$ : Length of the initial transition

$r_{2}$ : Radius waveguide prior to the first corrugation $d_{\text {imp }}:$ Depth of the first corrugation

$t_{i}: \quad$ Initial tooth thickness

$b_{i}$ : Initial slot thickness

$r_{3}$ : Final radius of the waveguide of the impedance adapter

$d_{0}$ : Nominal depth of slot

$t_{f}$ : Nominal tooth thickness

$b_{f}$ : Nominal slot thickness

$l_{p}$ : Length of the profile section

a: $\quad$ Aperture radius

$a_{1}$ : Control point 1

$a_{2}$ : Control point 2

$a_{3}$ : Control point 3

$a_{4}$ : Control point 4

$a_{5}$ : Control point 5 .

\section{Competing Interests}

The authors declare that they have no competing interests.

\section{Acknowledgments}

The authors want to acknowledge partial funding from MINECO Grants (FIS2012-38160).

\section{References}

[1] A. Balklavs, "Ventspils radiotelescopes: history, parameters, possibilities," Baltic Astronomy, vol. 5, pp. 181-186, 1996.

[2] F. Tercero, S. López-Ruíz, J. A. López-Fernández, and J. M. Serna, "Feed system design of the $16 \mathrm{~m}$ Radiotelescope of Ventspils (Latvia)," Tech. Rep., Observatorio de Yebes, 2016.

[3] K. Deb, A. Pratap, S. Agarwal, and T. Meyarivan, "A fast and elitist multiobjective genetic algorithm: NSGA-II," IEEE Transactions on Evolutionary Computation, vol. 6, no. 2, pp. 182-197, 2002.

[4] G. Subashini and M. C. Bhuvaneswari, "Non-dominated particle swarm optimization for scheduling independent tasks on heterogeneous distributed environments," International Journal of Advances in Soft Computing and Its Applications, vol. 3, no. 1, pp. 1-17, 2011.

[5] SMT Consultancies Ltd Home, Smtconsultancies.co.uk, 2016, http://www.smtconsultancies.co.uk/products/axial/axial.php.

[6] A. D. Olver, A. D. Olver, P. J. B. Clarricoats, A. A. Kishk, and L. Shafai, "Corrugated horns," in Microwave Horns and Feeds, vol. 39 of IEEE Electromagnetics Waves Series, chapter 9, 1994.

[7] X. Zhang, "Design of conical corrugated feed horns for wide-band high-frequency applications," IEEE Transactions on Microwave Theory and Techniques, vol. 41, no. 8, pp. 1263-1274, 1993.

[8] A. D. Olver, "Design of profiled corrugated horns," IEEE Transactions on Antennas and Propagation, vol. 36, no. 7, pp. 936-940, 1988.

[9] J. Teniente, R. Gonzalo, and C. Del-Río, "Ultra-wide band corrugated Gaussian profiled horn antenna design," IEEE Microwave and Wireless Components Letters, vol. 12, no. 1, pp. 20-21, 2002. 
[10] H. Deguchi, M. Tsuji, H. Shigesawa, and S. Matsumoto, "A compact low-cross-polarization horn antenna with serpentineshaped taper," in Proceedings of the IEEE Antennas and Propagation Society International Symposium, vol. 2, pp. 320-323, July 2001.

[11] L. Lucci, "NURBS profiled corrugated circular horns," in Proceedings of the IEEE Antennas and Propagation Society International Symposium, vol. 1, pp. 161-164, Columbus, Ohio, USA, June 2003.

[12] L. Pappula and D. Ghosh, "Synthesis of thinned planar antenna array using multiobjective normal mutated binary cat swarm optimization," Applied Computational Intelligence and Soft Computing, vol. 2016, Article ID 4102156, 9 pages, 2016.

[13] A. E. Eiben and J. E. Smith, Introduction to Evolutionary Computing, Natural Computing Series, Springer, Berlin, Germany, 1st edition, 2003.

[14] J. D. Schaffer, Multiple objective optimization with vector evaluated genetic algorithms [Ph.D. thesis], Vanderbilt University, Nashville, Tenn, USA, 1984.

[15] C. A. Coello-Coello and G. B. Lamont, Applications of MultiObjective Evolutionary Algorithms, World Scientific, Singapore, 2004.

[16] C. A. Coello Coello, D. A. Van Veldhuizen, and G. B. Lamont, Evolutionary Algorithms for Solving Multi-objective Problems, vol. 5 of Genetic Algorithms and Evolutionary Computation, Kluwer Academic, New York, NY, USA, 2002.

[17] A. Zhou, B.-Y. Qu, H. Li, S.-Z. Zhao, P. N. Suganthan, and Q. Zhangd, "Multiobjective evolutionary algorithms: a survey of the state of the art," Swarm and Evolutionary Computation, vol. 1, no. 1, pp. 32-49, 2011.

[18] M. A. Panduro, D. H. Covarrubias, C. A. Brizuela, and F. R. Marante, "A multi-objective approach in the linear antenna array design," International Journal of Electronics and Communications, vol. 59, no. 4, pp. 205-212, 2005.

[19] S.-H. Yeung, H.-K. Ng, and K.-F. Man, "Multi-criteria design methodology of a dielectric resonator antenna with jumping genes evolutionary algorithm," AEU-International Journal of Electronics and Communications, vol. 62, no. 4, pp. 266-276, 2008.

[20] R. Sánchez-Montero, P. Lõpez-Espí, D. Manjarres, I. LandaTorres, S. Salcedo-Sanz, and J. Del Ser, "Efficient design of a double-band coplanar hybrid antenna using multi-objective evolutionary programming," International Journal of Numerical Modelling: Electronic Networks, Devices and Fields, vol. 26, no. 6, pp. 620-629, 2013.

[21] K. M. Miettinen, Nonlinear Multiobjective Optimization, Kluwer Academic, Boston, Mass, USA, 1999.

[22] X. Yao, Y. Liu, and G. Lin, "Evolutionary programming made faster," IEEE Transactions on Evolutionary Computation, vol. 3, no. 2, pp. 82-102, 1999.

[23] D. E. Goldberg, Genetic Algorithms in Search, Optimization and Machine Learning, Addison-Wesley, Reading, Mass, USA, 1989.

[24] T. Bäck and H. P. Schwefel, "An overview of evolutionary algorithms for parameter optimization," Evolutionary Computation, vol. 1, no. 1, pp. 1-23, 1993.

[25] N. Srinivas and K. Deb, "Muiltiobjective optimization using nondominated sorting in genetic algorithms," Evolutionary Computation, vol. 2, no. 3, pp. 221-248, 1994.

[26] J. Kennedy and R. Eberhart, "Particle swarm optimization," in Proceedings of the 1995 IEEE International Conference on Neural Networks, pp. 1942-1948, December 1995.
[27] R. Sánchez-Montero, P. L. Lopez-Espí, A. C. Cruz-Rodriguez, and J. M. Rigelsford, "Multiple band antenna optimization using heuristics and bio-inspired optimization algorithms," in Proceedings of the Antennas and Propagation Conference (LAPC '12), pp. 1-4, Loughborough, UK, November 2012.

[28] V. Zuniga, A. Erdogan, and T. Arslan, "Control of adaptive rectangular antenna arrays using particle swarm optimization," in Proceedings of the 6th Loughborough Antennas and Propagation Conference (LAPC '10), pp. 385-388, Loughborough, UK, November 2010.

[29] M. Reyes-Sierra and C. A. Coello Coello, "Multi-objective particle swarm optimizers: a survey of the state-of-the-art," International Journal of Computational Intelligence Research, vol. 2, no. 3, pp. 287-308, 2006.

[30] P. S. Kildal, Foundations of Antennas, A Unified Approach, chapter 2, Student Literature, 2000.

[31] J. M. Serna, F. Tercero, T. Finn, and J. A. López, "The CDT ultra wide-band anechoic chamber," in Proceedings of the 5th European Conference on Antennas and Propagation (EUCAP '11), pp. 670-674, Rome, Italy, April 2011. 


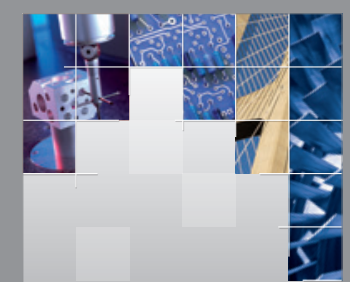

\section{Enfincering}
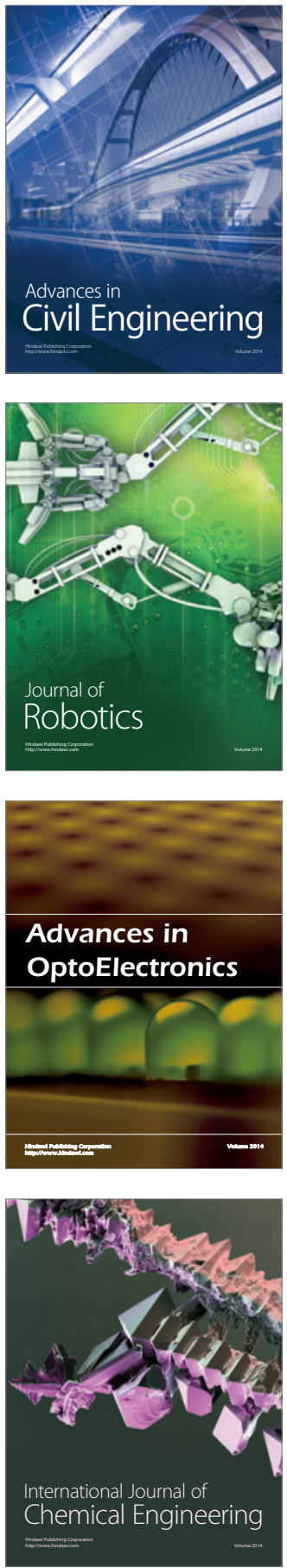

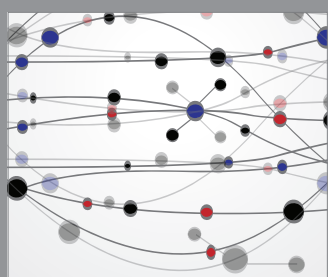

The Scientific World Journal

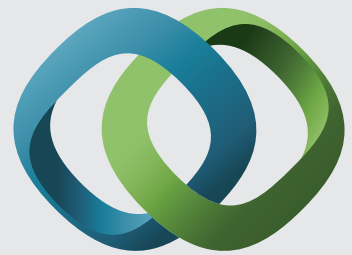

\section{Hindawi}

Submit your manuscripts at

http://www.hindawi.com
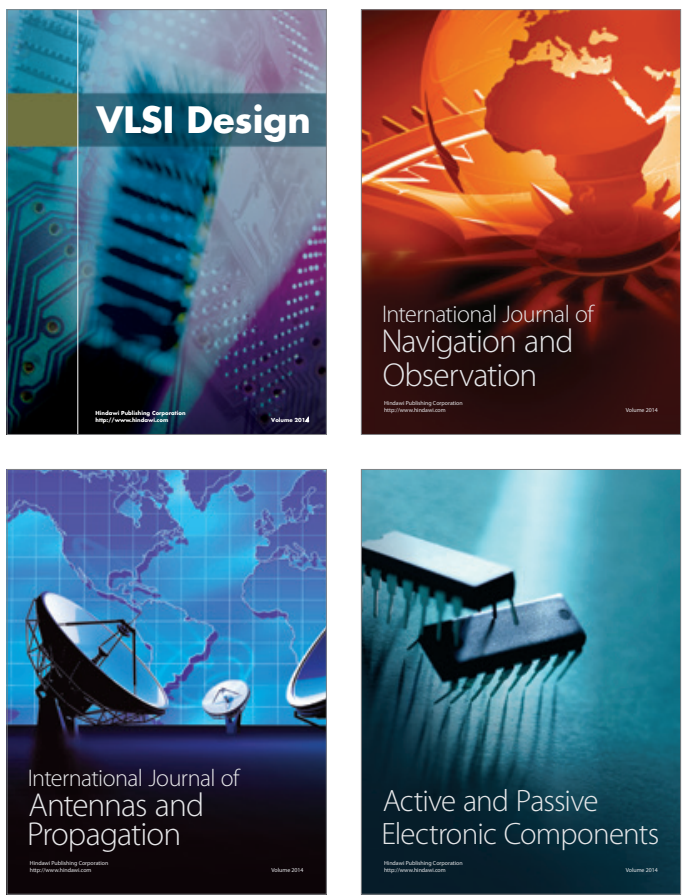
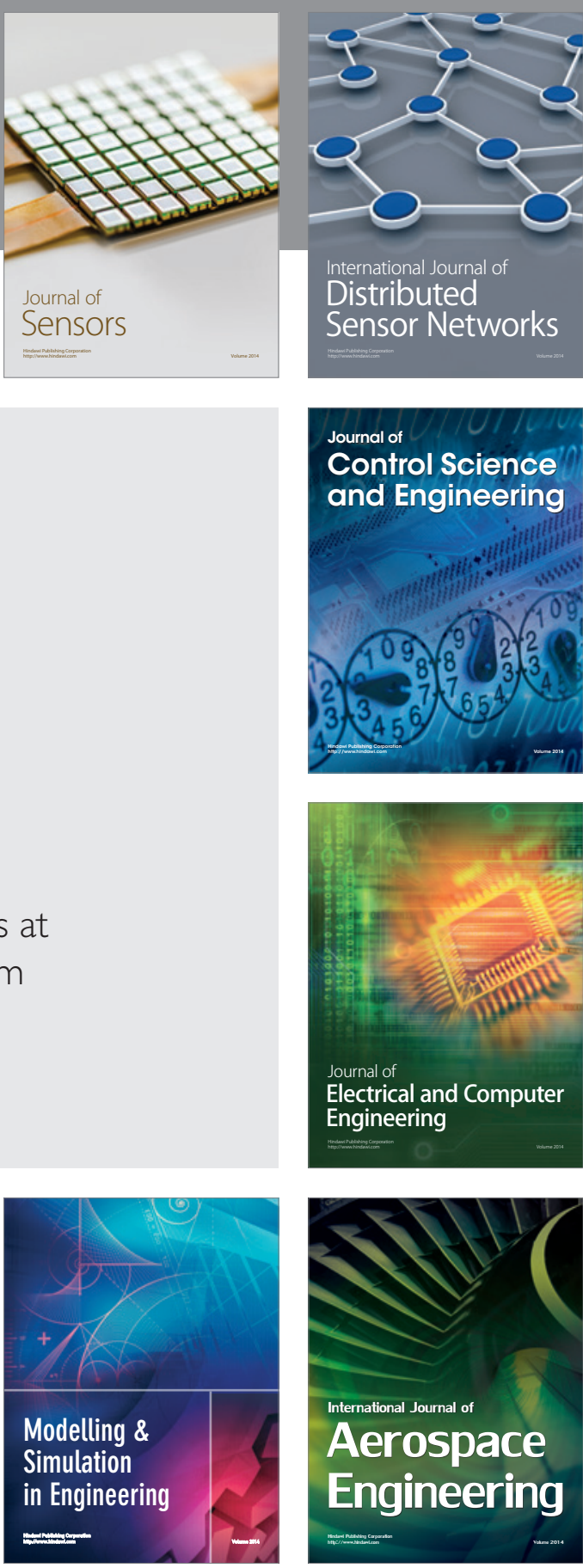

International Journal of

Distributed

Sensor Networks

Journal of

Control Science

and Engineering
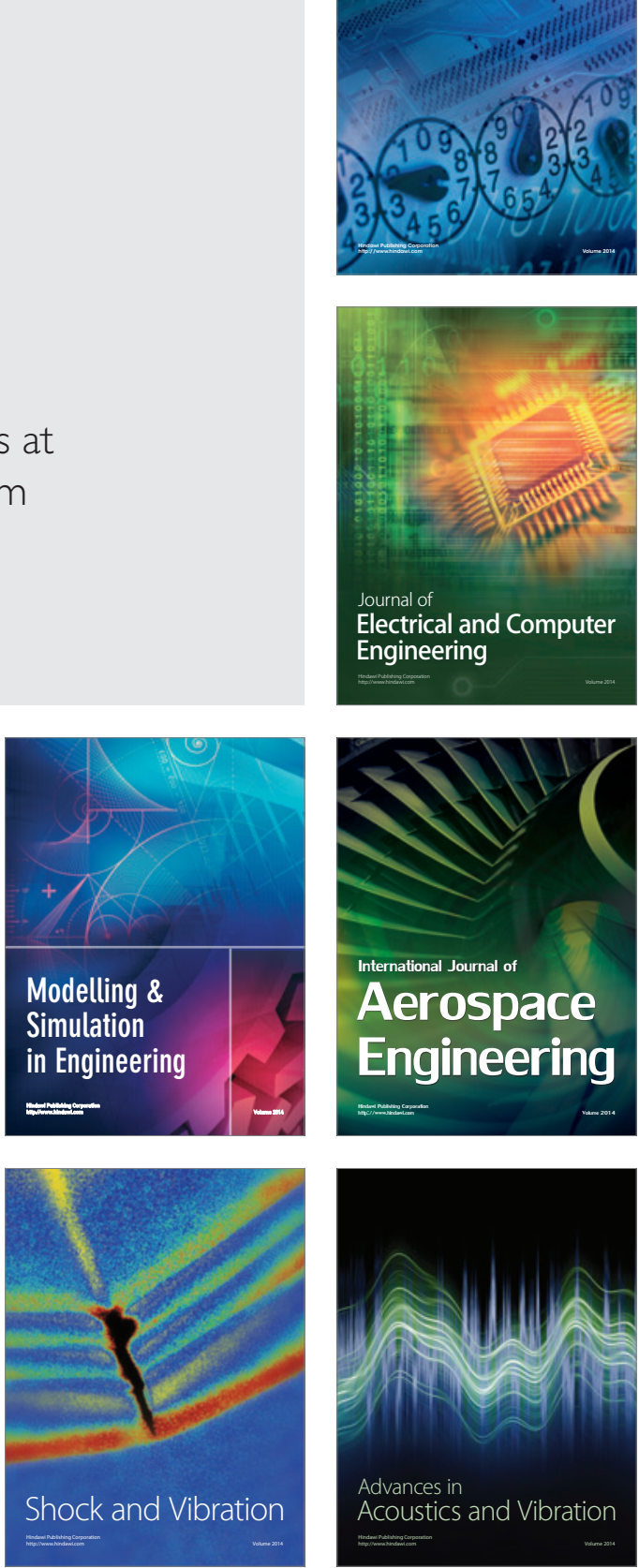